\section{KOMPASS}

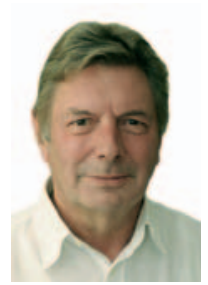

Prof. Dr. Gebhard Mathis

gebhard.mathis @ cable.vol.at

In den vergangenen Jahren hat die Thoraxsonographie beträchtliche Fortschritte erzielt. Zahlreiche hochkarätige Arbeiten sind erschienen - sowohl Multicenter-Studien als auch Meta-Analysen. Die Teilnehmer einer großen und sehr gründlichen internationalen Konsensuskonferenz im Jahr 2011, bei der 300 Arbeiten gesichtet wurden, erarbeiteten etliche differenzierte, aber vorsichtige Empfehlungen. Dabei wurde auch um eine einheitliche, klare Terminologie gerungen und das Wirrwarr von Abkürzungen, «signs» und Syndromen deutlich vereinfacht [1].

Die Einsatzmöglichkeiten des Ultraschalls in der Lungenheilkunde sind heute vielgestaltig: So sind mittels dieser Technologie inzwischen mehr als neun von zehn ambulant erworbenen Pneumonien mit einer Spezifität von 97\% darstellbar [2].

Ein Pneumothorax kann mithilfe der Sonographie unter Anwendung der erarbeiteten Kriterien mit hoher Treffsicherheit (im Vergleich zum Röntgenthorax) nachgewiesen oder ausgeschlossen werden [3] (deutsche Übersetzung siehe S. 75). Um das Ausmaß eines Pneumothorax darzustellen, braucht es allerdings weiterhin radiologische Verfahren. Doch allein die Ausschlussmöglichkeit ist bei der Diffe-

\section{Referenzen}

1 Volpicelli G, Elbarbary M, Blaivas M, Lichtenstein DA, Mathis G, Kirkpatrick AW, Melniker L, Gargani L, Noble VE, Via G, Dean A, Tsung JW, Soldati G, Copetti R, Bouhemad B, Reissig A, Agricola E, Rouby JJ, Arbelot C, Liteplo A, Sargsyan A, Silva F, Hoppmann R, Breitkreutz R, Seibel A, Neri L, Storti E, Petrovic T; International Liaison Committee on Lung Ultrasound (ILC-LUS) for International Consensus Conference on Lung Ultrasound (ICC-LUS): Inter-

\title{
Ultraschallstethoskop - die Zeit ist reif!
}

rentialdiagnose der Dyspnoe schon viel wert.

Durch die Entwicklung hochauflösender portabler Ultraschallsysteme ist es fast überall möglich, mit dem Gerät zum Patienten zu gehen und ihn bettseitig zu untersuchen, wie es im Rahmen der klinischen Abklärung mit dem Stethoskop geschieht. Es gibt verschiedenste technische Ausführungen, von der Pocket- über die Tablet- bis zur Laptop-Variante, zu durchaus erschwinglichen Preisen. Die beiden Letzteren können in der Auflösung mit High-End-Geräten mithalten. Durch raschere Diagnosen am Krankenbett, die Einsparung weiterer Untersuchungen und weniger Patiententransporte amortisieren sich diese Investitionen schnell.

Die Einführung der Mehrschicht-Computertomographie (MSCT) hat die Diagnostik der Lungenembolie revolutioniert. Zwar erhöhte sich dadurch die Zahl von Embolie-Diagnosen, jedoch konnte die Mortalität nicht signifikant gesenkt werden. Wird die Diagnose «Lungenembolie» also durch den Einsatz der MSCT bei Patienten gestellt, die gar keiner Therapie bedürfen? Mit dem Konzept der Multiorgan-Sonographie (Sensitivität 90-92\%) können wir eine Lungenembolie dagegen vergleichbar sicher darstellen; wahrscheinlich lassen sich damit zudem potentiell fatale Formen der Erkrankung rechtzeitiger diagnostizieren [4]. national evidence-based recommendations for point-of-care lung ultrasound. Intensive Care Med 2012;38:577-591.

2 Reissig A, Copetti R, Mathis G, Mempel C, Schuler A, Zechner P, Aliberti S, Neumann R, Kroegel C; Hoyer H: Lung ultrasound in the diagnosis and follow-up of community-acquired pneumonia: a prospective, multicenter, diagnostic accuracy study. Chest 2012;142:965-972.
Im letzten Jahrzehnt hat die endoluminale Sonographie das Staging des Lungenkarzinoms entscheidend verbessert [5] (deutsche Übersetzung siehe S. 68). Sie ist in den Leitlinien entsprechend platziert und in den pneumologischen Zentren auf hohem Niveau etabliert.

Doch im Hinblick auf die transkutane Thoraxsonographie erstaunt es, dass viele Pneumologen sich auf die sonographische Darstellung von Pleuraergüssen beschränken und darüber hinaus auf radiologische Bilder zurückgreifen. Was für den Pneumologen früher die Durchleuchtung war, ist heute die Sonographie, die allerdings eine wesentlich größere diagnostische Aussagekraft hat. Professor Martin Brutsche (siehe S. 85) hat völlig Recht mit seiner Forderung, «die transthorakale Ultraschalltechnik in das Ausbildungscurriculum von Pneumologen» aufzunehmen.

Neben den interessanten Beiträgen zum Schwerpunkt «pneumologischer Ultraschall» kommen in dieser Ausgabe von Karger Kompass Pneumologie viele weitere praxisrelevante Themen zur Sprache. Überzeugen Sie sich selbst.

Eine anregende Lektüre wünscht

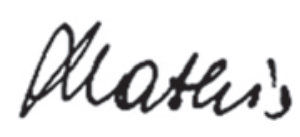

3 Kreuter M, Mathis G: Emergency ultrasound of the chest. Respiration 2014;87:89-97.

4 Mathis G: Thromboembolism in ultrasound: killing three birds with one stone. Chest 2014; 145:931-932.

5 Gompelmann D, Herth FJF: Role of endobronchial and endoscopic ultrasound in pulmonary medicine. Respiration 2014;87:3-8.

\section{KARGER \\ Fax +4976145207 14 \\ information@karger.com}

www.karger.com (c) 2014 S. Karger GmbH, Freiburg

2296-0368/14/0022-0066\$39.50/0

Accessible online at:

www.karger.com/kkp
Prof. Dr. Gebhard Mathis

Dr.-Summer-Straße 3

6830 Rankweil, Österreich 